\title{
Nuclear spin scissors mode - experimental status
}

\author{
Irina Molodtsova ${ }^{1, *}$, Evgeny Balbutsev ${ }^{1}$, and Peter Schuck $^{2}$ \\ ${ }^{1}$ Bogoliubov Laboratory of Theoretical Physics, Joint Institute for Nuclear Research, Dubna, 141980, Russia \\ ${ }^{2}$ Institut de Physique Nucléaire, IN2P3-CNRS, Université Paris-Sud, F-91406 Orsay Cédex, France; \\ Laboratoire de Physique et Modélisation des Milieux Condensés, CNRS and Université Joseph Fourier, 25 avenue des Martyrs BP166, \\ F-38042 Grenoble Cédex 9, France
}

\begin{abstract}
A new type of nuclear collective motion - the spin scissors mode - was predicted seven years ago. Promising signs of its existence in ${ }^{232} \mathrm{Th}$ were found. We perform a systematic analysis of experimental data on $M 1$ excitations in rare-earth nuclei to find traces of the spin scissors mode in this area. Obvious signs of its existence are demonstrated.
\end{abstract}

\section{Introduction}

The nuclear scissors mode was predicted by R. R. Hilton [1] as a counter-rotation of protons against neutrons in deformed nuclei. This idea was developed in the works of T. Suzuki, D. J. Rowe [2], N. Lo Iudice, F. Palumbo [3] and other authors and confirmed experimentally with its detection in ${ }^{156} \mathrm{Gd}$ by the group of A. Richter [4].
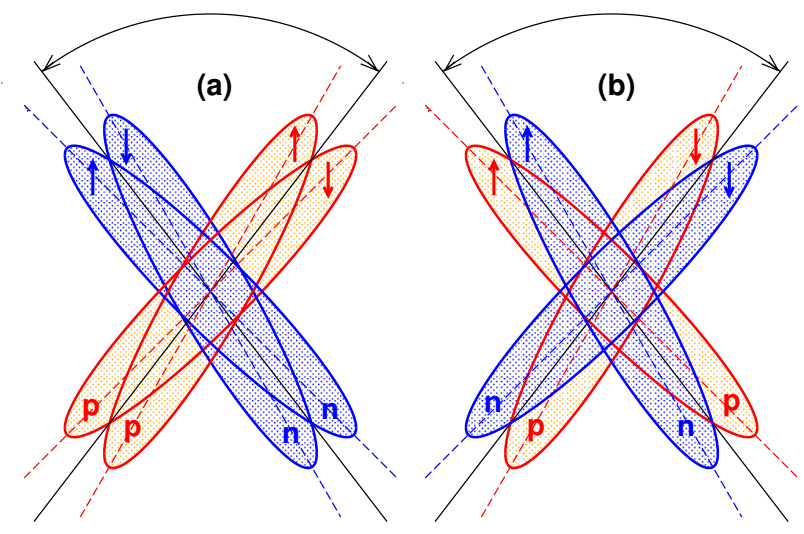

Figure 1. (a) - the orbital scissors (neutrons versus protons), (b) - spin scissors (spin-up nucleons versus spin-down nucleons). Arrows inside of ellipses show the direction of spin projections. $\mathrm{p}-$ protons, $\mathrm{n}-$ neutrons.

The Wigner Function Moments (WFM) method predicts a new type of nuclear collective motion - the spin scissors mode: rotational oscillations of nucleons with spin projection "up" with respect of "spin-down" nucleons. The pictorial view of these two different scissors is shown in figure 1.

A generalization of the WFM method which takes into account spin degrees of freedom and pair correlations si-

\footnotetext{
*e-mail: molod@theor.jinr.ru
}

multaneously was outlined in [5]. As a result the agreement between theory and experiment in the description of nuclear scissors modes was improved considerably.

The aim of the present work - to find the experimental confirmation of the splitting of low-lying $(E<4 \mathrm{MeV})$ $M 1$ excitations in two groups corresponding to spin and orbital scissors. It turns out that the splitting of low-lying $M 1$ excitations in two groups was observed and discussed by experimentalists already at the beginning of the "scissors era". For example, we cite from the paper of Wesselborg et al. [6]: "The existence of the two groups poses the question whether they arise from one mode, namely from scissors mode, or whether we see evidence for two independent collective modes."

\section{Brief description of the WFM method}

The basis of our method is the Time Dependent HartreeFock (TDHF) equation for the one-body density matrix $\rho^{\tau}\left(\mathbf{r}_{1}, \mathbf{r}_{2}, t\right)=\left\langle\mathbf{r}_{1}\left|\hat{\rho}^{\tau}(t)\right| \mathbf{r}_{2}\right\rangle:$

$$
i \hbar \frac{\partial \hat{\rho}^{\tau}}{\partial t}=\left[\hat{h}^{\tau}, \hat{\rho}^{\tau}\right]
$$

where $\tau$ is an isotopic index.

With the help of Fourier (Wigner) transformation the density matrix $\rho^{\tau}\left(\mathbf{r}_{1}, \mathbf{r}_{2}, t\right)$ is transformed into the Wigner function $f^{\tau}(\mathbf{r}, \mathbf{p}, t)$ and equation (1) is transformed into TDHF equation for $f^{\tau}(\mathbf{r}, \mathbf{p}, t)$.

Let us consider the simple Hamiltonian - a harmonic oscillator with separable quadrupole-quadrupole residual interaction:

$$
\begin{aligned}
& H=\sum_{i=1}^{A}\left(\frac{\mathbf{p}_{i}^{2}}{2 m}+\frac{1}{2} m \omega^{2} \mathbf{r}_{i}^{2}\right) \\
& +\sum_{\mu=-2}^{2}(-1)^{\mu}\left\{\bar{\kappa} \sum_{i}^{Z} \sum_{j}^{N}+\frac{\kappa}{2}\left[\sum_{i \neq j}^{Z}+\sum_{i \neq j}^{N}\right]\right\} q_{2 \mu}\left(\mathbf{r}_{i}\right) q_{2-\mu}\left(\mathbf{r}_{j}\right),
\end{aligned}
$$


Table 1. Scissors modes energies $E$ and transition probabilities $B(M 1)$ for ${ }^{164} \mathrm{Dy}$. The results of calculations [9] with and without the spin part of a dipole magnetic operator $\left(g_{s}=0.7 g_{s}^{\text {free }}\right.$ and $g_{s}=0$ respectively).

\begin{tabular}{|c|c|c|c|c|c|c|}
\hline \multirow[b]{2}{*}{${ }^{164}$ Dy } & \multicolumn{3}{|c|}{$E, \mathrm{MeV}$} & \multicolumn{3}{|c|}{$B(M 1), \mu_{N}^{2}$} \\
\hline & $\begin{array}{c}\text { spin } \\
\text { scissors }\end{array}$ & $\begin{array}{c}\text { orbital } \\
\text { scissors }\end{array}$ & centroid & $\begin{array}{c}\text { spin } \\
\text { scissors }\end{array}$ & $\begin{array}{c}\text { orbital } \\
\text { scissors }\end{array}$ & $\Sigma$ \\
\hline$g_{s}=0.7 g_{s}^{\text {free }}$ & 2.77 & 3.60 & 3.07 & 2.44 & 1.36 & 3.80 \\
\hline$g_{s}=0$ & 2.77 & 3.60 & 3.50 & 0.76 & 6.10 & 6.86 \\
\hline
\end{tabular}

where $q_{2 \mu}=\sqrt{16 \pi / 5} r^{2} Y_{2 \mu}$ and $N, Z$ are the numbers of neutrons and protons respectively.

Integrating the equation for the Wigner function over phase space with the weights

$$
\{r \otimes p\}_{\lambda \mu},\{r \otimes r\}_{\lambda \mu},\{p \otimes p\}_{\lambda \mu},
$$

where $\{r \otimes p\}_{\lambda \mu}=\sum_{\sigma, v} C_{1 \sigma, 1 v}^{\lambda \mu} r_{\sigma} p_{v}$ one gets dynamic equations for second order moments

$$
\begin{aligned}
& R_{\lambda \mu}^{\tau}(t)=\frac{2}{(2 \pi \hbar)^{3}} \int d \mathbf{p} \int d \mathbf{r}\{r \otimes r\}_{\lambda \mu} f^{\tau}(\mathbf{r}, \mathbf{p}, t), \\
& P_{\lambda \mu}^{\tau}(t)=\frac{2}{(2 \pi \hbar)^{3}} \int d \mathbf{p} \int d \mathbf{r}\{p \otimes p\}_{\lambda \mu} f^{\tau}(\mathbf{r}, \mathbf{p}, t), \\
& L_{\lambda \mu}^{\tau}(t)=\frac{2}{(2 \pi \hbar)^{3}} \int d \mathbf{p} \int d \mathbf{r}\{r \otimes p\}_{\lambda \mu} f^{\tau}(\mathbf{r}, \mathbf{p}, t),
\end{aligned}
$$

which play the role of the collective variables. Physical meaning of these variables: $R_{2 \mu}$ - quadrupole moment of the system and $R_{00}$ - mean square radius, $P_{2 \mu}$ and $P_{00}-$ quadrupole moment and mean square radius in a momentum space. The variables $L_{\lambda \mu}$ describe the coupling of momentum and coordinate spaces, $L_{1 \mu}$ being the orbital angular momentum. As a result, we obtain a system of coupled dynamical nonlinear equations for protons $(\tau=\mathrm{p})$ and neutrons $(\tau=\mathrm{n})$ :

$$
\begin{array}{ll}
\frac{d}{d t} R_{\lambda \mu}^{\tau}-\frac{2}{m} L_{\lambda \mu}^{\tau}=0, & \lambda=0,2 \\
\frac{d}{d t} L_{\lambda \mu}^{\tau}-\frac{1}{m} P_{\lambda \mu}^{\tau}+m \omega^{2} R_{\lambda \mu}^{\tau} & \\
-12 \sqrt{5} \sum_{j=0}^{2} \sqrt{2 j+1}\left\{\begin{array}{ll}
11 j \\
2 \lambda 1
\end{array}\right\}\left\{Z_{2}^{\tau} \otimes R_{j}^{\tau}\right\}_{\lambda \mu}=0, & \lambda=0,1,2 \\
\frac{d}{d t} P_{\lambda \mu}^{\tau}+2 m \omega^{2} L_{\lambda \mu}^{\tau} & \\
-24 \sqrt{5} \sum_{j=0}^{2} \sqrt{2 j+1}\left\{{ }_{2 \lambda 1}^{11 j}\right\}\left\{Z_{2}^{\tau} \otimes L_{j}^{\tau}\right\}_{\lambda \mu}=0, & \lambda=0,2
\end{array}
$$

where $\left\{\begin{array}{l}11 j \\ 2 \lambda 1\end{array}\right\}$ is the Wigner $6 j$-symbol. The functions $Z_{2 \mu}^{\mathrm{n}}=\kappa R_{2 \mu}^{\mathrm{n}}+\bar{\kappa} R_{2 \mu}^{\mathrm{p}}$ and $Z_{2 \mu}^{\mathrm{p}}=\kappa R_{2 \mu}^{\mathrm{p}}+\bar{\kappa} R_{2 \mu}^{\mathrm{n}}$ implement the coupling of neutron and proton equations.

By definition [1] the scissors mode is the pure isovector mode. That is why dynamical equations are usually transformed into isovector and isoscalar ones. These equations are linearized and decoupled in a small amplitude approximation. In this case it is possible to solve them analytically, the solution being identical with that of RPA [7, 8].

We are interested in the scissors mode with quantum number $K^{\pi}=1^{+}$. Therefore, we need only a part of isovector dynamical equations with $\mu=1$. Imposing the time evolution via $\mathrm{e}^{i \Omega t}$ for all variables one transforms the system of dynamical equations into a set of algebraic equations. Eigenfrequencies are found as solutions of its secular equation. It is necessary to emphasize that all conservation laws and sum rules are fulfilled.

Taking into account pair correlations and spin degrees of freedom allows one to get the satisfactory agreement with experiment in the description of energies and $B(M 1)$ values of the scissors mode [5].

Both scissors modes have an underlying orbital nature, because both are generated by the same type of collective variables - by the orbital angular momenta.The difference is that the "orbital" scissors are generated by the counter-oscillations of the orbital angular momentum of proton body with respect of the orbital angular momentum of neutron body, whereas the "spin" scissors are generated by the counter-oscillations of the orbital angular momentum of all nucleons with spin projection "up" with respect of the orbital angular momentum of all nucleons with spin projection "down". At the same time, the results of calculations with and without the spin part of a dipole magnetic operator $\left(g_{s}=0.7 g_{s}^{\text {free }}\right.$ and $g_{s}=0$ respectively $)$ show that both scissors are equally sensitive to the spin dependent part of nuclear forces, demonstrating the moderate constructive interference of the orbital and spin contributions in the case of the spin scissors mode and their very strong destructive interference in the case of the orbital scissors mode (see table 1).

\section{Experimental situation}

Let us analyze the experimental data in search of indications of splitting. We have in mind the possible concentration of the observed $1^{+}$excitations in two more or less separated groups, the lower group being stronger than higher one.

\subsection{Actinides}

A very clear cut example of splitting is observed in ${ }^{232} \mathrm{Th}$, see figure 2 . The spectrum of ${ }^{232} \mathrm{Th}$ can be divided in two groups with certainty. For the Uranium isotopes the separation of spectrum into two groups is not that obvious. To exclude any arbitrariness in their division we apply a simple technical device. We folded the experimental spectra with a Lorentzians of increasing width. The width was increased until only two humps remained. The obtained lower and higher groups of levels are attributed to the spin and orbital scissors modes respectively. Comparing their 
Table 2. Energy centroids $E$ and summed transition probabilities $B(M 1)$ of the spin and orbital scissors. Exp. - experimental data: ${ }^{232} \mathrm{Th}-[10],{ }^{236} \mathrm{U}-[11],{ }^{238} \mathrm{U}-[12]$. WFM - the results of calculations [9], the values of all model parameters are taken from our paper [5].

\begin{tabular}{|c|c|c|c|c|c|c|c|c|c|c|c|c|}
\hline \multirow{3}{*}{ Nuclei } & \multicolumn{6}{|c|}{$E, \mathrm{MeV}$} & \multicolumn{6}{|c|}{$B(M 1), \mu_{N}^{2}$} \\
\hline & \multicolumn{2}{|c|}{ spin scissors } & \multicolumn{2}{|c|}{ orbital scissors } & \multicolumn{2}{|c|}{ centroid } & \multicolumn{2}{|c|}{ spin scissors } & \multicolumn{2}{|c|}{ orbital scissors } & \multicolumn{2}{|l|}{$\sum$} \\
\hline & Exp. & WFM & Exp. & WFM & Exp. & WFM & Exp. & WFM & Exp. & WFM & Exp. & WFM \\
\hline${ }^{232} \mathrm{Th}$ & 2.15 & 2.30 & 2.99 & 2.93 & 2.49 & 2.54 & $2.52(26)$ & 2.40 & $1.74(38)$ & 1.42 & $4.26(64)$ & 3.82 \\
\hline${ }^{236} \mathrm{U}$ & 2.10 & 2.33 & 2.61 & 2.96 & 2.33 & 2.57 & $2.01(25)$ & 2.83 & $1.60(26)$ & 1.73 & $3.61(51)$ & 4.56 \\
\hline${ }^{238} \mathrm{U}$ & 2.19 & 2.36 & 2.76 & 3.00 & 2.58 & 2.61 & $2.46(31)$ & 3.18 & $5.13(89)$ & 2.02 & $7.59(1.20)$ & 5.20 \\
\hline
\end{tabular}

energy centroids and summed $B(M 1)$ values with theoretical predictions we find very good agreement for ${ }^{232} \mathrm{Th}$, satisfactory agreement in ${ }^{236} \mathrm{U}$ (figure 3 ), and a rather conditional one in ${ }^{238} \mathrm{U}$ (table 2) [9].

\subsection{Rare earths}

We have studied practically all papers containing experimental data for low-lying $M 1$ excitations in rare-earth nuclei. For the sake of convenience we have collected in figure 4 all experimentally known spectra of low-lying $1^{+}$ excitations (see paper [13] and references therein). One glance on figure 4 is enough to understand that the situation with spectra in rare-earth nuclei is complicated. Nevertheless, we will try to proceed in the same way as with the actinides: we fold the spectra with a Lorentzian whose width is increased until only two humps survive, if possible. The states in the low-lying group are then identified as belonging to the spin scissors motion and the ones of high-lying group as orbital scissors states.

The values of $B(M 1)$ and energy centroids, corresponding to the described separation, are compared with theoretical results in figure 5. For energy centroids, the picture looks quite acceptable. The distribution of the scissors mode strength between spin and orbital states, at first glance, does not seem convincing. However, with closer examination, encouraging examples of agreement can be

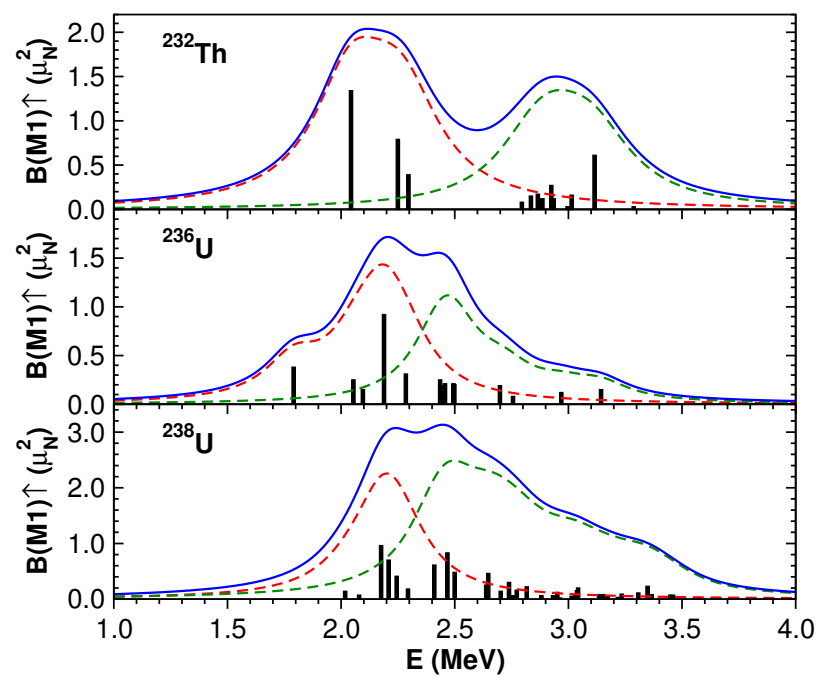

Figure 2. The experimentally observed spectra of $1^{+}$excitations: ${ }^{232} \mathrm{Th}-[10],{ }^{236} \mathrm{U}-[11],{ }^{238} \mathrm{U}-[12]$.

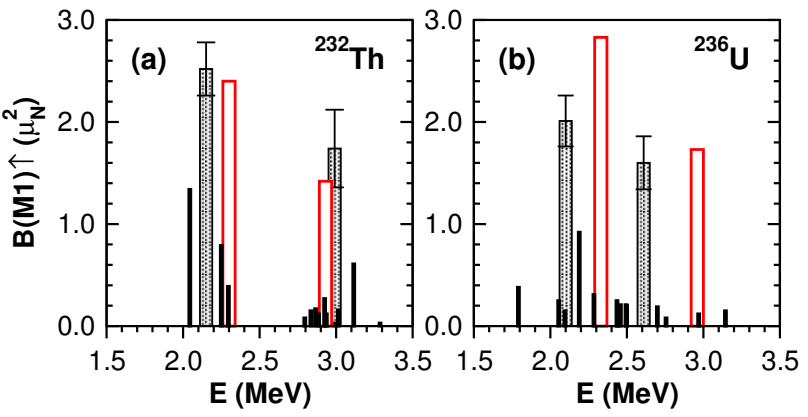

Figure 3. The centroids of experimentally observed spectra of $1^{+}$ excitations in ${ }^{232} \mathrm{Th}$ and ${ }^{236} \mathrm{U}$ (black rectangles with error bars) are compared with the results of WFM calculations (red rectangles) of the spin and orbital scissors modes [9].

found. Satisfactory (as a rule, within the experimental error) agreement between theoretical and experimental results was found for the orbital scissors in 13 nuclei: ${ }^{146,148} \mathrm{Nd},{ }^{154} \mathrm{Gd},{ }^{166,170} \mathrm{Er},{ }^{174,176} \mathrm{Yb},{ }^{176,178,180} \mathrm{Hf},{ }^{190} \mathrm{Os}$ and ${ }^{194,196} \mathrm{Pt}$. The same degree of agreement can be found for the spin scissors in 15 nuclei: ${ }^{134} \mathrm{Ba},{ }^{146,148,150} \mathrm{Nd}$, ${ }^{150,154} \mathrm{Sm},{ }^{154} \mathrm{Gd},{ }^{160} \mathrm{Dy},{ }^{172,174,176} \mathrm{Yb},{ }^{176,180} \mathrm{Hf},{ }^{192} \mathrm{Os}$ and ${ }^{194} \mathrm{Pt}$. An agreement for both, the orbital and spin scissors, is observed in 8 nuclei: ${ }^{146,148} \mathrm{Nd},{ }^{154} \mathrm{Gd},{ }^{174,176} \mathrm{Yb}$, ${ }^{176,180} \mathrm{Hf}$ and ${ }^{194} \mathrm{Pt}$. As can be seen from the figure 6, the agreement in $\mathrm{Yb}$ and $\mathrm{Hf}$ isotopes is close to the perfect one.

\section{Conclusions and Outlook}

The aim of this paper was to find experimental indications about the existence of the "spin" scissors mode predicted in our previous publications $[5,14,15]$. To this end we have performed the detailed analysis of experimental data on $1^{+}$excitations for actinides and for nuclei of $N=82-126$ major shell.

We have found good quantitative agreement between the theory and experiment for the summed $B(M 1)$ values and the respective energy centroids for the majority of nuclei except for some few. The best agreement is obtained when one attributes to the scissors mode all $1^{+}$excitations observed in the energy interval $0<E<4 \mathrm{Mev}$ (excluding separate levels in some nuclei). It turns out also that the results of our calculations [16] are in very good agreement with the sum rule derived in [13].

For the fine structure of the scissors mode in rare-earth nuclei satisfactory (mostly very good) agreement between 

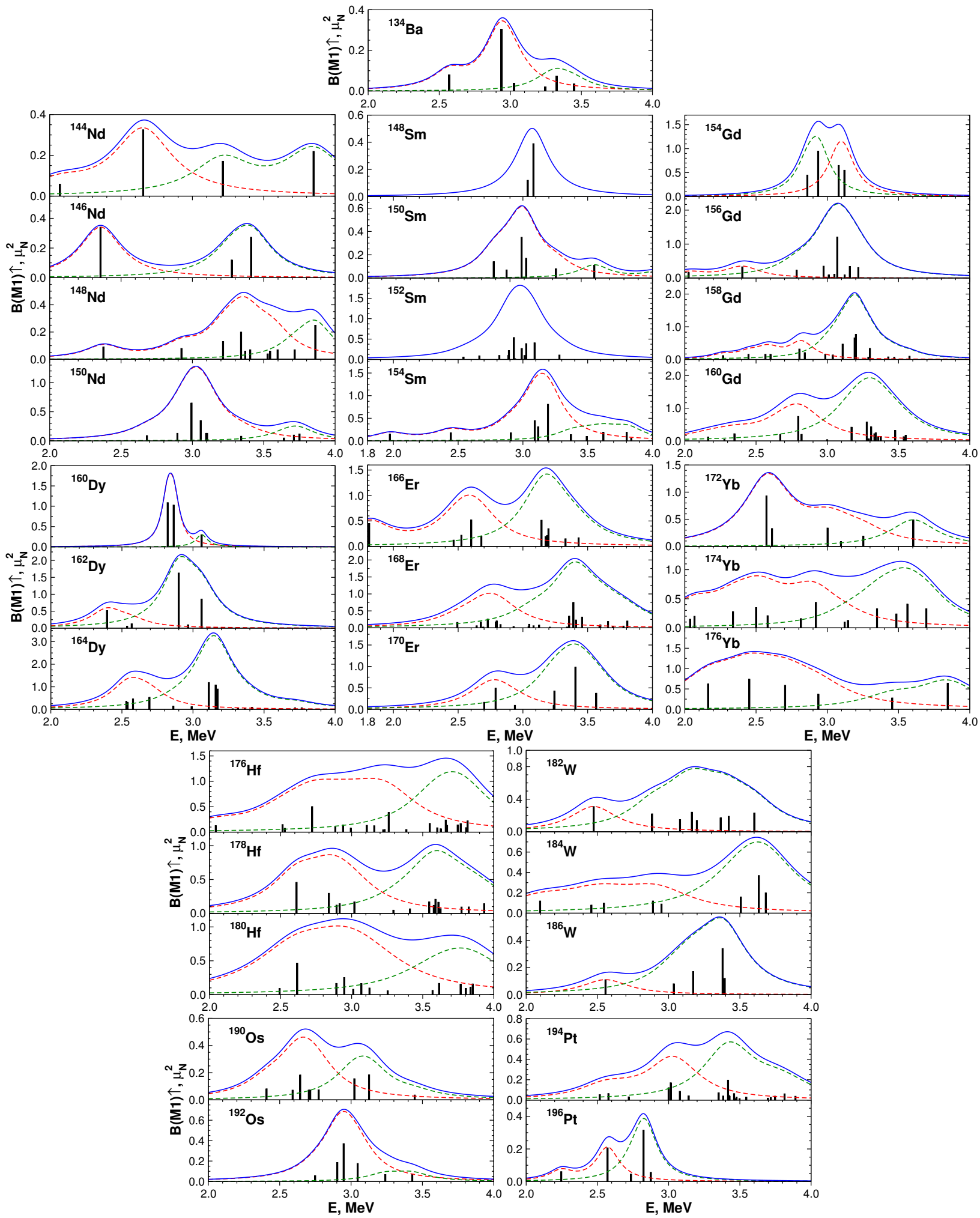

Figure 4. The experimentally observed spectra of $1^{+}$excitations. The data are taken from references listed in table 1 of the paper [13]. 

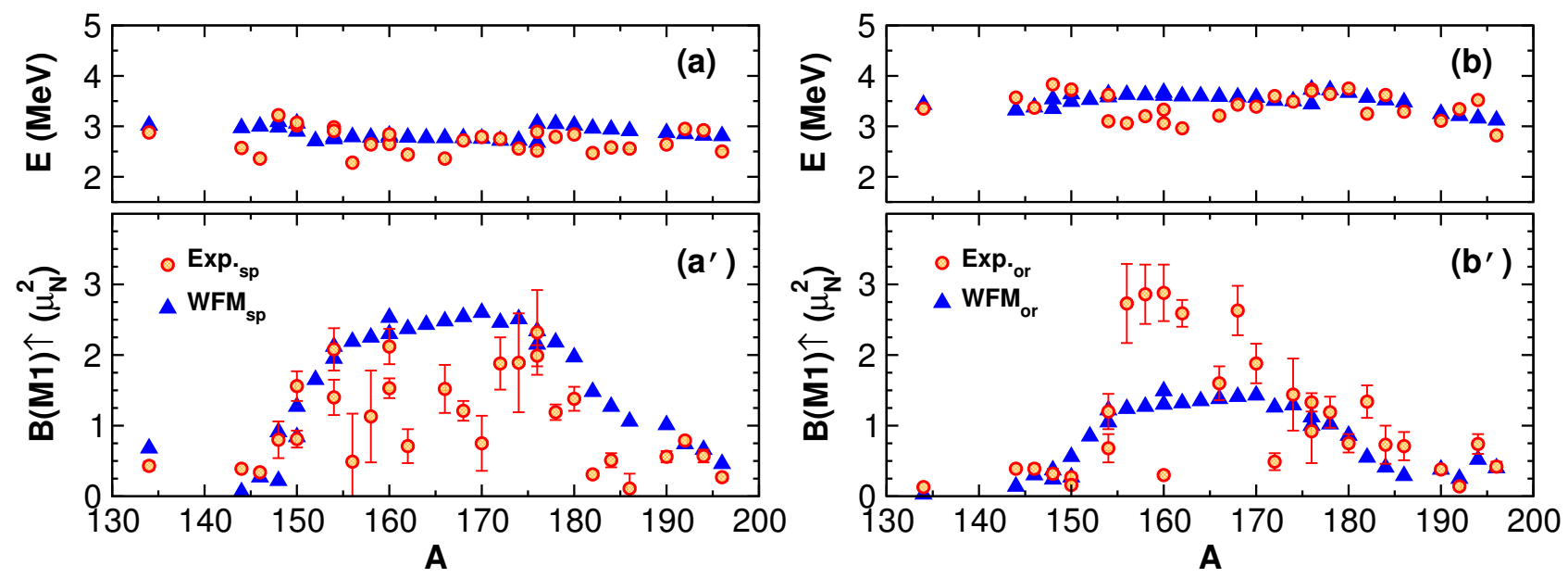

Figure 5. Energies $E(\mathrm{a}, \mathrm{b})$ and transition probabilities $B(M 1)\left(\mathrm{a}^{\prime}, \mathrm{b}^{\prime}\right)$ of the spin scissors and the orbital scissors obtained by dividing experimental spectra into two groups. $\mathrm{WFM}_{\mathrm{sp}}$ and $\mathrm{WFM}_{\mathrm{or}}$ - the results of calculations [9] for spin and orbital scissors respectively;

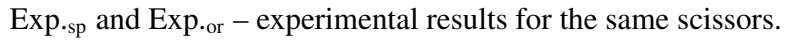

the theory and experiment is observed in 8 nuclei. In the rest of the nuclei the situation is uncertain and requires further theoretical (and, possibly, experimental) research. In actinides very good agreement is observed for ${ }^{232} \mathrm{Th}$, satisfactory agreement in ${ }^{236} \mathrm{U}$, and a rather conditional one in ${ }^{238} \mathrm{U}$ [9].

It is shown that both scissors are strongly influenced by the spin dependent part of nuclear forces, so the observed $1^{+}$states having a spin nature may also be attributed to the scissors mode.

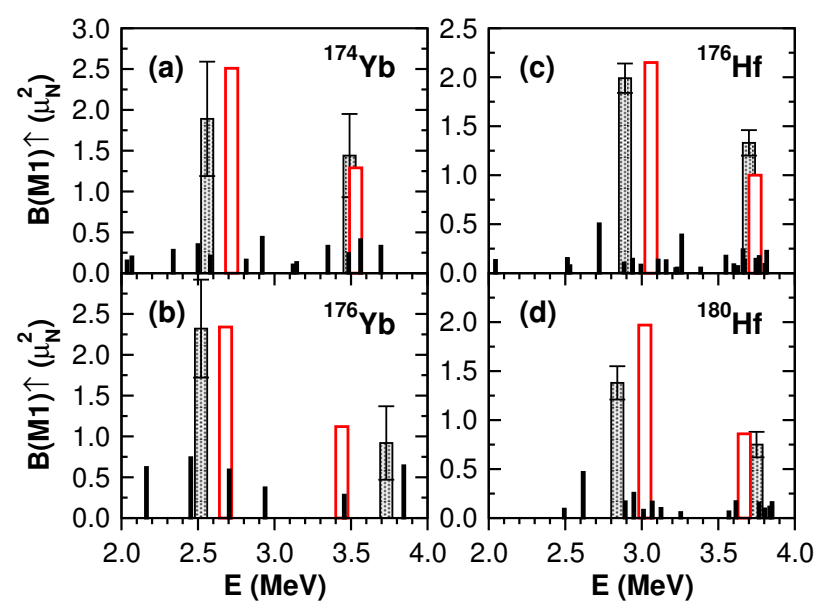

Figure 6. The centroids of experimentally observed spectra of $1^{+}$excitations in ${ }^{174,176} \mathrm{Yb}(\mathrm{a}, \mathrm{b})$ and ${ }^{176,180} \mathrm{Hf}(\mathrm{c}, \mathrm{d})$ (black rectangles with error bars) are compared with the results of calculations [9] (red rectangles) of the spin and orbital scissors modes. Notice that the strength of the lower peak (spin-scissors) is always stronger than the one of the upper peak (orbital-scissors).

To gain the comprehensive insight into the fine structure of the scissors mode we are going to perform the calculations with fourth order moments which, as we hope, will allow us to expand substantially the set of input data for the WFM method and to study the dynamics of the spin-flip resonance and its possible influence on the scissors mode. Furthermore the systematic self consistent QRPA calculations with realistic forces are very desirable. We already said $[14,15]$ that such excitations (spin scissors) were, undoubtedly, produced implicitly by other methods (e.g. RPA), but they never were analyzed in such terms. So it would be interesting to perform the analysis of QRPA spectra analogously to our analysis of experimental spectra. At least one example [17] of QRPA calculations exists, demonstrating rather good agreement with experimental data and with our results for ${ }^{232} \mathrm{Th}$.

Based on the presented analysis of the accumulated experimental information, it can be concluded that the existence of a spin (together with a spin-orbit interaction) leads to a splitting of the scissors mode into two branches corresponding to spin and orbital scissors.

\section{References}

[1] R. R. Hilton, Talk presented at the International Conference on Nuclear Structure (Joint Institute for Nuclear Research, Dubna, Russia, 1976) (unpublished)

[2] T. Suzuki and D. J. Rowe, Nucl. Phys. A 289, 461 (1977)

[3] N. Lo Iudice and F. Palumbo, Phys. Rev. Lett. 41, 1532 (1978)

[4] D. Bohle, A. Richter, W. Steffen, A. E. L. Dieperink, N. Lo Iudice, F. Palumbo, and O. Scholten, Phys. Lett. B 137, 27 (1984)

[5] E. B. Balbutsev, I.V. Molodtsova, and P. Schuck, Phys. Rev. C 91, 064312 (2015)

[6] C. Wesselborg, P. von Brentano, K. O. Zell, R. D. Heil, H. H. Pitz, U. E. P. Berg, U. Kneissl, S. Lindenstruth, U. Seemann, and R. Stock, Phys. Lett. B 207, 22 (1988)

[7] E. B. Balbutsev and P. Schuck, Nucl. Phys. A 720, 293 (2003); 728, 471 (2003) 
[8] E. B. Balbutsev and P. Schuck, Ann. Phys. 322, 489 (2007)

[9] E. B. Balbutsev, I.V. Molodtsova, and P. Schuck, Phys. Rev. C 97, 044316 (2018)

[10] A. S. Adekola, C. T. Angell, S. L. Hammond, A. Hill, C. R. Howell, H. J. Karwowski, J. H. Kelley, and E. Kwan, Phys. Rev. C 83, 034615 (2011)

[11] J. Margraf, A. Degener, H. Friedrichs, R. D. Heil, A. Jung, U. Kneissl, S. Lindenstruth, H. H. Pitz, H. Schacht, U. Seemann, R. Stock, C. Wesselborg, P. von Brentano, and A. Zilges, Phys. Rev. C 42, 771 (1990)

[12] S. L. Hammond, A. S. Adekola, C. T. Angell, H. J. Karwowski, E. Kwan, G. Rusev, A. P. Tonchev,
W. Tornow, C. R. Howell, and J. H. Kelley, Phys. Rev. C 85, 044302 (2012)

[13] J. Enders, P. von Neumann-Cosel, C. Rangacharyulu, and A. Richter, Phys. Rev. C 71, 014306 (2005)

[14] E. B. Balbutsev, I.V. Molodtsova, and P. Schuck, Nucl. Phys. A 872, 42 (2011)

[15] E. B. Balbutsev, I.V. Molodtsova, and P. Schuck, Phys. Rev. C 88, 014306 (2013)

[16] E. B. Balbutsev, I.V. Molodtsova, and P. Schuck, Yad. Fiz. 81 (Iss. 5), 1 (2018)

[17] A. A. Kuliev, E. Guliyev, F. Ertugral, and S. Özkan, Eur. Phys. J. A 43, 313 (2010) 